\title{
Volumen 2

Cona -Juego del Hilo Sin Fin- en las prácticas de maternaje de las Madres Cuidadoras de la Cultura Qom. Indagaciones en perspectiva de educación intercultural en contextos sociales con pueblos indígenas

\section{Cona-Game of the Endless Thread-in the mothering practices of the Caregiving Mothers of the Qom Culture. Inquiries from the perspective of intercultural education in social contexts with indigenous peoples}

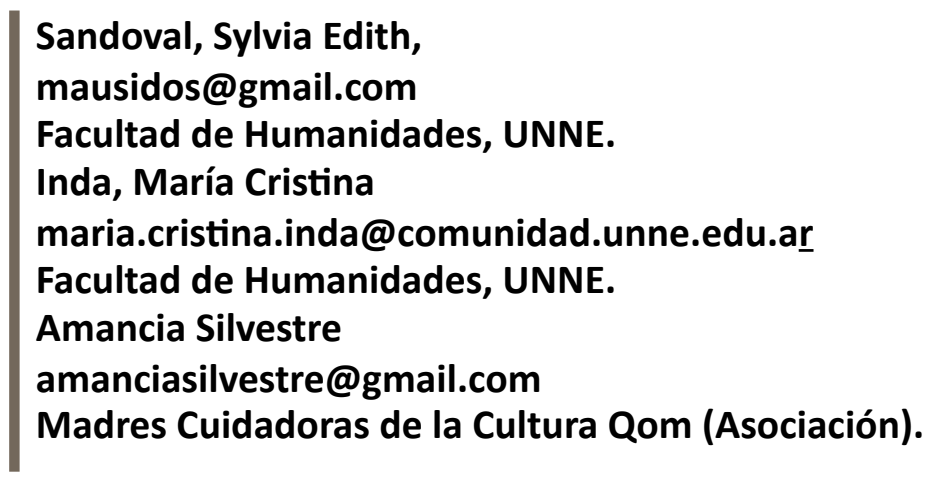

Sandoval, Sylvia Edith es Profesora en Historia (UNNE), Mgter. en Epistemología y metodología de la Investigación Científica (UNNE), Doctora en Filosofía (UNNE). Adjunta a/C de la asignatura Ciencias Sociales en la Educación Inicial del Profesorado y Licenciatura en Educación Inicial, Facultad de Humanidades, (UNNE). Directora del proyecto de Investigación Resol. 098/19. "Las Prácticas de Maternaje Qom y su aporte a la formación de grado en Educación Bilingüe Intercultural en contextos sociales con Pueblos Indígenas. Cuarta Etapa”.

Inda, María Cristina es Profesora en Biología (UNNE), Licenciada en Botánica (UNNE), Especialista en Enseñanza de las Ciencias (FLACSO), Magister en Gestión Ambiental (UNNE), Profesora Titular de la asignatura Ciencias Naturales en la Educación Inicial del Profesorado y Licenciatura en Educación Inicial, Facultad de Humanidades, (UNNE). Jefa de Trabajos Prácticos en la asignatura Biogeografía y Geografía Ambiental del Profesorado y Licenciatura en Geografía, Facultad de Humanidades, (UNNE). Co-Directora proyecto de Investigación Resol. 098/19. "Las Prácticas de Maternaje Qom y su aporte a la formación de grado en Educación Bilingüe Intercultural en contextos sociales con Pueblos Indígenas. Cuarta Etapa". Profesorado y Licenciatura en Educación Inicial, Facultad de Humanidades, (UNNE).

Amancia Silvestre es Pedagoga Idónea de la Cultura Qom, Profesora de la asignatura Cultura y artesanías indígenas del Profesorado en Educación Inicial en el Centro Educación Superior Bilingüe Intercultural -CESBI- de Pampa del Indio. Ministerio de Educación de la provincia del Chaco. Integrante del PI 18H004. Las Prácticas de Maternaje Qom y su aporte a la formación de grado en Educación Bilingüe Intercultural en contextos sociales con Pueblos Indígenas. Cuarta Etapa. Resol. 098/19 (13-Marzo). (01-01-2019 al 31-12-22). 


\section{| Resumen}

El presente trabajo comunica uno de los temas trabajados en el proyecto de investigación ${ }^{1}$ sobre las prácticas de maternaje de las Madres Cuidadoras de la Cultura Qom²,específicamente sobre Cona Juego del Hilo Sin Fin-. En las culturas por lo general el juego ocupa un lugar importante, tanto como la necesidad lúdica propia de Homo ludens (J.Huizinga: 1938) como de la función social que cumple. En las prácticas de maternaje de las MCCQ el juego es imprescindible para el desarrollo de destrezas en las/os niñas/os qom: adiestrarse a trepar árboles, correr, esconderse, imitar a los animales, silbar, gruñir, mimetizarse, entre otras habilidades que les permiten convivir en el ambiente natural a la vez que desarrollar la identidad cultural mediada por la lengua materna y la pertenencia al territorio. Cona -Juego del Hilo Sin Fin- como prefieren traducirlo las MCCQ comparte como juego que es, la infinita posibilidad de representar el mundo de la vida y las cosas que lo habitan. En el presente trabajo analizaremos y reflexionaremos en clave interdisciplinar sobre algunos atributos de Cona vinculados al trabajo de memoria y su relación con la historia, como su aporte a la etnobiología. Hay que mencionar que las características que adquiere Cona provienen de un análisis compartido sostenido en un marco de referencia de diálogo entre culturas y contextualizado en perspectiva intercultural, necesaria para la formación de grado en contextos sociales con pueblos indígenas.

\section{| Palabras claves}

Prácticas de maternaje - Cultura Qom- Cona -memoria - historia

\section{| Abstract}

This paper communicates one of the topics worked on in the research project on the practices of the Caregiving Mothers of the Qom Culture, specifically on Cona -Juego del Hilo Sin Fin-. In cultures, play usually occupies an important place, both as the playful need of Homo ludens (J.Huizinga: 1938) and as the social function it fulfills. In the mothering practices of the MCCQ, play is essential for the development of skills in Qom children: training to climb trees, run, hide, imitate animals, whistle, growl, mimic, among other skills that They allow them to coexist in the natural environment while developing cultural identity mediated by their mother tongue and belonging to the territory. Cona - Game of the Endless Thread - as the MCCQ prefer to translate it, shares as a game that it is, the infinite possibility of representing the world of life and the things that inhabit it. In this paper we will analyze and reflect in an interdisciplinary way on some attributes of Cona linked to memory work and its relationship with history, such as its contribution to ethnobiology. It should be mentioned that the characteristics acquired by Cona come from a shared analysis sustained in a framework of dialogue between cultures and contextualized in an intercultural perspective, necessary for undergraduate training in social contexts with indigenous peoples.

\section{| Keywords}

Practices of the Caregiving Mothers- Qom Culture- Cona - memory - history

$1 \quad$ PI 18H004. Las Prácticas de Maternaje Qom y su aporte a la formación de grado en Educación Bilingüe Intercultural en contextos sociales con Pueblos Indígenas. Cuarta Etapa. Resol. 098/19 (13-Marzo). (01-01-2019 al 31-12-22)

2 En adelante MCCQ. 


\section{Volumen 2 \\ "Semiótica y Narrativas"}

\section{Artículo}

Revista del grupo de

Estudios SEMTO-DISCURSNOS

juego de lenguaje en el reino de la memoria, pues nos relaciona con el 'pasado' de nuestra comunidad, y con ello, también, re-articulan nuestra historicidad (como pertenencia y rememoración)" (García, 2004, Pp. 214-215).

Desde la perspectiva intercultural que precede este estudio, Cona constituye una ventana por la que miramos e interpretamos significados que vienen de la historia oral de un tiempo ancestral y de un tiempo reciente.

La interculturalidad se presenta como un proceso de participación interactivo en el que son precisamente los sujetos y sus prácticas los que están en juego y que, por eso, son éstos los llamados a la interpretación de lo intercultural, pero como sujetos implicados y no como objetos observados. (Fornet Betancourt, 2005).

La interpretación desde la interculturalidad se realiza desde un contexto histórico real, en un tiempo y espacio particular. Numerosos e importantes estudios provenientes de distintos campos disciplinares, pero particularmente de la antropología, dan cuenta de la importancia del juego del hilo para interpretar la cosmovisión de los pueblos indígenas. La relación con el cielo, con los ciclos naturales, con lo sobrenatural, con la subsistencia diaria se expresa detalladamente en las figuras del juego del hilo. Entramos al mundo de los objetos y de las relaciones entre los sujetos y los objetos, por lo que se refiere a ello, la transmisión oral en lengua materna o lata' qaq cobra importancia en el correlato entre la memoria y la historia oral.

Bajo el actual régimen eminentemente lingüicida y para evitarles el sufrimiento a sus hijos, cada vez más padres indígenas les hablan a sus hijos en la lengua hegemónica. Cambio también motivado por el deseo de facilitar su acceso a la educación formal y, en general, al empleo y a la vida citadina, ya que ésta es mediada únicamente por la lengua europea. Por tanto, se ha hecho bastante común que el proceso de socialización primaria sea vehiculado por la lengua hegemónica, incluso cuando los padres solo tengan un dominio parcial de ella. Si bien esto se da con más frecuencia en las zonas urbanas, el fenómeno se está extendiendo gradualmente a las zonas rurales, debido al rápido avance de la economía de mercado, del consumismo y del capitalismo. (López, 2015, p. 220).

Los Qom, como los demás pueblos indígenas del Chaco, son de tradición oral. Por esta razón, Cona se realiza narrando historias y relatos en qom, de este modo la memoria, las destrezas de las manos y el gesto corporal se revitalizan, la lengua se incorpora performativamente en la vida diaria recreando y practicando aquellas costumbres que deben cuidarse porque en ellas están las claves de su cosmovisión y las estrategias para el cambio.

Estamos expuestos al lenguaje antes que cualquier posible constitución o representación de un acto de habla. Entonces podremos ver cómo el acto del habla nos afecta y anima en nuestros cuerpos: el campo de la susceptibilidad y el afecto es ya un registro corporal de algún tipo. (Butler, 2017, p. 69 )

\section{| 1.2. Prácticas de maternaje y el valor de Cona como registro de la memoria de la comunidad.}

Si bien la recuperación de la memoria es un atributo cognitivo y práctico (Ricoeur, 2008, p. 82) 


\section{Volumen 2

en zonas de contacto entre culturas y por lo general con tensiones y conflictos de intereses históricos, su uso social adquiere un propósito político. El caso de las MCCQ es uno de los ejemplos de recuperación de la lengua madre y de la memoria ancestral, son hablantes activos/as de la lengua qom y del español, como ciudadanas y conscientes de ello, organizaron una asociación civil étnica ${ }^{3}$ que, junto a otras organizaciones qom de Pampa del Indio, toman decisiones importantes para la comunidad. Cona, Juego del Hilo Sin Fin- da cuenta de la vitalidad de la lengua qom en la transmisión de la socialización primaria o prácticas de maternaje de las MCCQ.

Las prácticas de maternaje corresponden a la madre y deben ser aprehendidas y transmitidas de modo individual y comunitario a las hijas e hijos desde que nacen hasta los 10 años aproximadamente. Expresan las interacciones de todos los integrantes de la comunidad, conformando la trama del tejido social con facultades, limitaciones y obligaciones. La lengua, el tiempo comunitario vivido, el mito, los relatos, los preceptos y las enseñanzas, los tabúes, la familia, el cuidado de los hijos, la alimentación, los juegos, los espacios y los lugares son entre otras, sus expresiones materiales. Los bienes culturales transmitidos a través de las prácticas de maternaje se sostienen en la memoria y el territorio vivido, las prácticas culturales y el entorno natural y los juegos ancestrales. (Sandoval et al., 2013, Pp. 50-51)

Cona comienza y recomienza entre la evocación del pasado y la actualización del presente. Narraciones y relatos ancestrales ejercitan las destrezas del trabajo de memoria sostenido en la práctica de la historia oral, transmitida de generación en generación. Cada figura de Cona puede narrar -por sí misma o en composición con otras- relatos de una historia compartida, de recuerdos que conducen al tiempo vivido en la comunidad. Estos relatos componen distintos tejidos narrativos que llevan impresiones de actividades diarias de la subsistencia como la recolección de frutos, la pesca, la caza, recreativas como juegos, celebraciones y aquellas vinculadas con el mito de la cosmología. El hilo de Cona enlaza huellas del pasado reciente con el pasado ancestral. Conviene subrayar el efecto práctico que adquiere la transmisión de Cona en las prácticas de maternaje y durante toda la vida. En tal sentido, resulta sugestivo pensar Cona como un habilitador de recuerdos de las historias de vida y de los relatos de las propias biografías que en su conjunto pueden constituir la propia historia de la comunidad. El juego, resulta un gran ejercicio de evocación para interpretar los significados de las extraordinarias experiencias vividas en el monte y en el vasto territorio del Gran Chaco.

Distintos relatos, procedentes del canon de las memorias individuales condensan en historias comunes y compartidas. Quienes cuentan estos relatos convertidos en historias de la comunidad tienen el respeto y la sabiduría para contar una historia contrahegemónica. Los hechos de esta historia alcanzan un tiempo inmemorial como las memorias del tiempo reciente, entre ambos, el dolor y la resistencia. En todo caso, se trata de experiencias ocurridas en el territorio-vivido por las propias MCCQ y transmitidas en lengua materna.

\section{| 2. Cona: la trama de la vida y la cultura que se entretejen y entrelazan}

“...Yo el Cháguar, fui testigo durante muchas generaciones, de cómo los mayores educaban a los jóvenes para el amor y la vida familiar. Mujeres y varones crecian en comunidades que les transmitían sus normas de conducta por medio de ritos sagrados. Ellos entonces llegaban a ser dueños de sus impulsos. Esto fue así mientras

3 Asociación Civil Qomlashepi Huo’o Naq Henec (Madres Cuidadoras de la Cultura Qom) Personería Jurídica Res. N 354/24/04/19, Inspección General de Personas Jurídicas y Registro Público de Comercio. Gobierno del Chaco 


\section{Volumen 2 \\ "Semiótica y Narrativas"}

\section{Artículo}

Revista del grupo de

Estudios SEMIO-DISCURSNOS

no tuvieron contacto con culturas distintas. Muchas manos femeninas tejieron mis fibras...las mujeres tenian mucha influencia en las decisiones del grupo, y algunas llegaron a tener autoridad reconocida. Y así, yo la modesta planta de Cháguar, y ellas, las mujeres autóctonas, tejíamos juntas los hilos de esta historia del Gran Chaco...".

("Memorias del Cháguar". Mercedes Silva (1998). Memorias del Gran Chaco. Segunda Parte. Edipen. Resistencia.)

Una mujer Qom aprende desde muy niña a tejer, a deslizar los dedos suavemente entre los hilos para armar la madeja que poco a poco irá transformándose en una nueva creación. El hilo es un símbolo, es un cordón sutil que une el paso de la historia a través de las generaciones, en una trama familiar y comunitaria. Cada tejedora es una incansable celebradora de la vida: en sus innumerables vueltas del telar o de la aguja, en cada lanzada o punto anudado ata la historia, anuda la cultura, preserva la memoria y siembra esperanza. La qom lashepi lleva estos hilos al mundo de arriba... entreteje, imagina redes nuevas, formando un tejido de re-unión entre el cielo y la tierra, los legados antiguos y las nuevas creaciones que siempre capturan la originalidad de cada artesana. Si algo le sale mal, desata con paciencia lo tejido y vuelve a comenzar lo mismo, insiste en cultivar la esperanza, sueña e imagina. El hilo nos habla y nos recuerda, invita a unir en prodigioso momento el pasado, el presente y el amplio porvenir. Así, en un devenir que se actualiza, la tejedora de alma despliega los hilos invisibles de las almas libres, fortalece la trama del pueblo y la memoria en un apretado empeño por soñar y construir un nuevo tejido social para todos y todas. La trama del amor, vivo hoy en estos rostros y estas manos, continúa...

Cháguar es el nombre común de un cardo nativo. El nombre es un vocablo de origen quechua que significa exprimir, estrujar, porque se la estruja para sacarle las fibras.

La denominación guaraní es caraguatá. Se denominan cháguar a varias especies relacionadas de plantas sudamericanas de la familia Bromeliaceae, entre ellas Bromelia serra, Bromelia hieronymi, Bromelia urbanianum y Pseudananas sagenarius que viven en el monte fuerte, especialmente en zonas conservadas y sin vestigios de contaminación alguna en la amplia región semi-árida del Gran Chaco Americano.

Las mujeres con sus hijos e hijas pequeños, salen al monte en grupos a recoger cháguar, por caminos polvorientos, que sortean entre vegetación espinosa, soportando el calor de una larga caminata de muchos kilómetros. Parte del ritual comienza con este compartir grupal y la solidaridad de hacerlo entre compañeras.

Los chaguarales (agrupaciones de plantas de cháguar en el monte) representan un tesoro natural, particularmente las plantas del centro, ya que sus hojas y sus fibras son más largas y luego de un largo proceso que se va realizando en etapas, se obtiene el hilo, la fibra de la planta que se adquiere de las hojas, se tiñe con tintes naturales (a través de frutos, cortezas, raíces, hollín y otras fuentes tintóreas) y posteriormente se arma la madeja para ser tejida (Inda, 2013). El proceso completo desde la extracción de las hojas hasta el teñido y el hilado puede consultarse en el audiovisual en el sitio de la Universidad Nacional del Nordeste, referido al tema, que fue una producción audiovisual del trabajo colaborativo junto a las MCCQ.

El pueblo Qom habitaba un territorio amplio en el que encontraban todo lo necesario para su subsistencia, que era recorrido anualmente moviéndose según el ritmo de las estaciones que permitían la abundancia de frutos y semillas para elaborar harina, miel de abejas, cardúmenes de peces en las lagunas o animales silvestres que proveían de carne, cueros y/o plumas. 


\section{Volumen 2

Ester Silvestre 4 , cuenta "en este territorio vivían nuestros ancestros, el lugar era libre. Ellos se trasladaban de un lugar a otro, no habia alambrados, no habia blancos, sino que eran todos originarios del lugar. ellos se trasladaban de un lugar a otro y tenían una relación muy concreta con la naturaleza. el respeto era lo principal para todos. Respetaban al agua, al monte, porque su economía estaba basada en ese lugar. Comían diferentes tipos de carnes, de peces y de animales del monte como ser carpincho, guazuncho, tapir, todos diferentes tipos de animalitos que viven en el monte. Iguana, tatú, tatú mulita, yacaré, hay infinito tipo de animalitos que servía para consumo. Cuando ellos veían que los animales o la pesca se estaba agotando se trasladaban a otro lugar. Todo lo que conseguía se compartía”.

Los Qom constituyen un pueblo que vive y transcurre su vida en relación con la naturaleza, percibiéndose parte de esa madre pródiga que hace crecer los seres que comparten su existencia dependiendo unos de otros. Nos cuenta una anciana qom, una de las fundadoras de las Madres Cuidadoras de la Cultura Qom de Pampa del Indio, Margarita, que "los antepasados tenían más contacto con el monte, con el río. Por eso es que adoraban, cuidaban. Pedían permiso antes de entrar al monte, antes de entrar al río porque el monte tiene sus protectores, hay espíritus que protegen el monte, el rio y las lagunas".

El topónimo monte que puede asimilarse al término paisajístico de bosque alto es utilizado por los Qom para designar el tipo de formación vegetal que provee al pueblo todo lo necesario, sería similar a un supermercado o una farmacia, nos explica Aureliana, quien actualmente es la coordinadora de las MCCQ. La gente comía peces, carpincho, yacaré, miel. Y después comía tuna, porque había tuna en el campo y con eso vivía la gente. Después comía ananá en el campo y en la costa del rio. Se comía mistol y algarroba, continúa en su narración.

El monte fuerte presenta quebrachos colorados, quebrachos blancos, palo lanza, guaraninás, espinas corona, virarós, en una intrincada trama de lianas y enredaderas, orquídeas y helechos y en cuyo sotobosque crecen en bajo los rayos filtrados del sol, los cardos o chaguar que los qom utilizan para tejer. El Gran Chaco americano es uno de los biomas que alberga la mayor biodiversidad de la biosfera, la vida vegetal en sus múltiples formas, sirve de cobijo y hogar para la fauna con que se relacionaron desde tiempos antiguos estas sociedades indígenas que lo habitaron antes de la llegada del conquistador europeo. El pueblo qom transcurre la vida insertos en su entorno natural, con todos los seres del monte, plantas, animales, sol, agua. La vida de cada integrante de la comunidad Qom: cada mujer, hombre, niña o niño sólo puede explicarse a partir de esta íntima relación con todas las formas de vida que pueblan el monte. Crecen juntos, se entrelazan en un ciclo vital, se acompañan, creando una profunda relación de sociedad humana y naturaleza, en armonía y equilibrio natural. Son generalmente las mujeres las principales depositarias de este conocimiento natural, son ellas las conocedoras idóneas de los diferentes usos y modos de utilización de hojas, plantas, raíces, flores, semillas, frutos, grasa de animales, cenizas, maderas, como tesoros escondidos en el mundo natural de nuestro Chaco. La mujer es el ser privilegiado que está en contacto con la vida por esencia, la maternidad natural y espiritual que ejerce cuidando la vida en todas sus formas, de forma respetuosa y cercana, se evidencia en su relación privilegiada con los saberes etnobiológicos. Durante el transcurrir de su vida como hija recibe generalmente de sus ancestras, abuelas o madre el conocimiento delicado y respetuoso en la familia, que constituirá su fuente de saberes cuando le toque desempeñarse como esposa y madre.

La etnobiología busca conocer y desentrañar esta relación tan antigua y respetuosa. Quiere acercarse al misterio que encierra este encuentro, lugar de sabiduría ancestral, fuente de saberes milenarios transmitidos de generación en generación en las tramas familiares y comunitarias. El Cona 
o juego del hilo va anudando los hilos entrelazando las formas de la naturaleza en la destreza de los dedos que siguen recreando la cultura y nombrando los vocablos antiguos. El juego del hilo sin fin- Cona- recibe este nombre particular pues abre un espacio casi hasta el infinito en asociar las representaciones de figuras, tomadas del entorno cercano y a partir de la detallada observación se representan en forma simbólica a través del lenguaje que escriben los dedos de las manos en las fibras de cháguar. Actualmente, también ha venido con las nuevas formas de vida el reemplazo del material original, del hilado de las fibras de las hojas espinosas y largas del cháguar por otros como el algodón de variados colores o la lana, que son sucedáneos del hilo ancestral, tejido a partir del valioso cardo.

El juego valoriza la memoria y sirve de ejercitación práctica para estimular los movimientos de las manos, la coordinación entre los ojos y el movimiento, a la vez que adiestra el pensamiento abstracto para representar en una forma un elemento concreto natural. Las formas de los astros en el cielo, los cuerpos de los peces en su entorno de agua y los animales o partes de sus cuerpos son las figuras que van desentrañando un contacto de seres que habitan juntos y son parte de un mismo sitio, el lugar que sustenta toda la trama de la vida. La vida familiar que refuerza los lazos sanguíneos transcurre dando lugar a actividades comunitarias que incluyen la recreación y el juego entre niños, niñas, jóvenes y adultos, padres y parientes. Se juega y comparte en comunidad, especialmente cuando el día va terminando dando paso al atardecer, a la vez que se ríe y disfruta hablando el qom laqtaq en un distendido clima de aprendizaje, pues siempre va acompañado de narraciones en voz suave y con competencias que incentivan estimulando las destrezas viso-motoras de los más pequeños. Los niños y niñas aprovechan a compartir jugando en los patios de las escuelas donde de la mano de las y los maestros (que los niños llaman "tía", dándole una entidad de perteneciente a su familia), recuperan estas formas de aprendizaje, consolidándose en la memoria de los sentidos.

Si se considera la semiosfera (Lotman: 1996) como un espacio de sentido que a modo de urdimbre va tejiendo significados contextualizados, la etnobotánica como la etnomatemática -disciplina que estudia las expresiones étnicas de este campo- generan espacios de sentido, en cuya trama se entrelazan conceptos, ideas, prácticas y valores provenientes del campo científico como de la cosmovisión qom en el caso de Cona. En esta línea de trabajo, las siguientes narraciones realizadas por las MCCQ van acompañadas de interpretaciones construidas en diálogo intercultural.

\section{| Amancia:}

-Hoy vamos a jugar el juego del hilo sin fin que es un juego autóctono de los pueblos originarios y desde tiempos milenarios se viene transmitiendo a las nuevas generaciones. Un juego con distintas figuras de animales, aves, también figuras de estrellas. Se llama juego del hilo sin fin porque se hacen las figuras contando de uno a diez en toba. Hasta el número diez existen las figuras o los números en etnomatemática qom. Nuestros antiguos no tenían la regla de matemática. Se lo llama hilo sin fin porque tiene muchísimas figuras que representan varias cosas con este hilo. Puede jugar cualquier niña o niño, es para los dos. La importancia de esto es hacer valorar la imaginación, tener habilidades en las manos y a la vez aprender. Basado en esto se trabaja con los números en la cosmovisión qom, en la etnomatemática qom nosotros relacionamos el lucero con el número uno, que es el primero que sale a la mañana y a la tarde. Después tenemos el numero dos que son dos cona, nosotros le llamamos así. La pata del ñandú es el número tres, también puede ser como tres cona lucero, el cuatro, el cinco es la figura del pescadito, hasta el nueve. La figura de la yica, el caramelo, las hojas del palo borracho o yuchán.

Cona del Lucero -Chi'Ishe

Se forma una ronda de narradoras, Victoria comienza en qom el relato. Amancia, traduce al 


\section{Volumen 2 \\ "Semiótica y Narrativas"}

\section{Artículo}

Revista del grupo de

Estudios SEMTO-DISCURSNNOS

español mientras Lucía dibuja entre sus dedos la figura del Lucero del amanecer o Chi'Ishe. Todas las narradoras realizan las figuras de Cona mientras hablan, como si estuvieran escribiendo el relato con las formas. Amancia: -

-Era la marisca, la pesca o ya sea actividades en la casa. Victoria había relatado que en época de invierno se lo ve muy resplandeciente y como que va cambiando de colores en tiempos de helada. Los tobas hacían los juegos con los hilos de cháguar para representar la figura del lucero en el amanecer. Este es un juego tradicional de los tobas, de todas las culturas indígenas -digamos-.

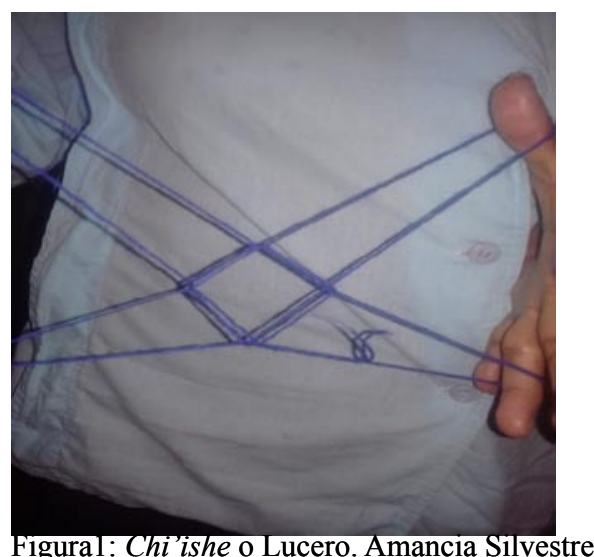

\section{| Amancia:}

-Lo que relata Victoria es que antiguamente era el horario de la madrugada cuando se tenían que levantar, veían si estaba el lucero, porque venía amaneciendo y para salir a pescar o a cazar nuestros antiguos tenían como horario al lucero.

Graciela:

-Ellos, una vez que veían esa estrella que sale, entonces se levantan, le pasa el sueño y ahí ellos se preparaban para hacer todas sus actividades en el día.

Desde la etnomatemática, el número 1 es el lucero, chi'she astro resplandeciente que es el primero en aparecer temprano en ese misterioso devenir que preanuncia el día, en las horas del alba. A esa hora los qom se levantaban antiguamente para hacer sus actividades, pues al ritmo de las horas de la luz se salía a mariscar (vocablo que se traduce como buscar en el monte o los humedales los recursos alimenticios o para satisfacer las necesidades), a pescar, a cazar o las mujeres a realizar sus actividades domésticas del hogar. El lucero es la primera estrella en el cielo por la mañana, pero también el primer astro brillante en el atardecer, presagiando el comienzo del tiempo nocturno. Sus colores indican signos que los ancianos enseñan anunciando tiempo frío con heladas.

El número 2, representa dos almejas de la laguna o el río. Son pequeños invertebrados que se incluyen en la dieta habitual del pueblo a la vez que brindan un caparazón calcáreo al que se trabaja en diversos usos.

El número 3, mañec, es la figura que remite a la pata del ñandú. Este es un animal que ha coevolucionado en los paisajes chaqueños de pastizales y praderas y tiene un gran valor de bien cultural que se evidencia en relatos ancestrales fundantes como el del Ñandú del cielo. La especie animal sirve pues de sus tendones de los miembros inferiores se elaboran tientos usados para coser sandalias, ropas. También se refiere un uso etnobiológico para alimentación, vestido y medicinas. Cona del Mañec lapia' -pata del Ñandú- 


\section{Volumen 2 \\ "Semiótica y Narrativas"}

\section{Artículo}

Revista del grupo de

Estudios SEMIO-DISCURSNOS

\section{Graciela:}

- Como sabemos, nuestros antepasados, nuestros abuelos, ellos cuando cazaban el ñandú le sacaban las venas para costurear sus ropas o para costurear sus sandalias y todo lo que ellos usaban. Después la carne, eso también ellos comían, las plumas, eso también guardaban para sus remedios y la grasa también ellos guardaban para su remedio. Por eso nosotros las figuras que hacemos, valoramos mucho para pasar a los nuevos, para que ellos vayan sabiendo todos los significados de lo que nosotros le enseñamos a ellos, le pasamos.

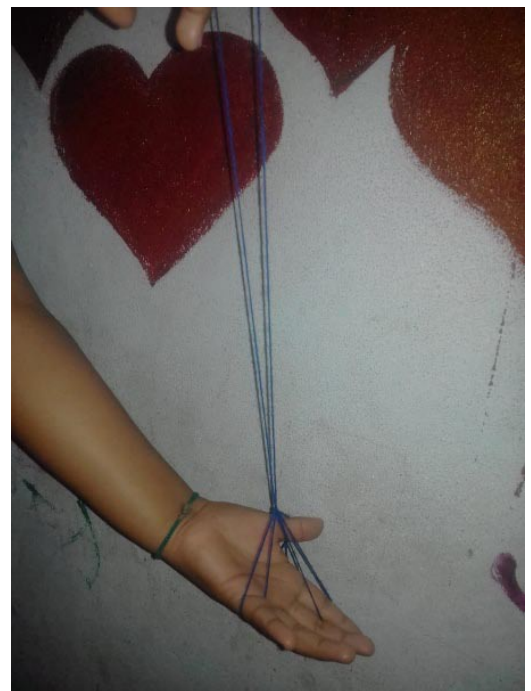

Figura 2: Mañec lapia’. Amancia Silvestre

El número 4, cona, son las almejas que viven en comunidad en los ríos y lagunas, en las zonas húmedas del Chaco. Son animales que el niño y la niña aprende desde su infancia a incorporar entre los vocablos en lengua materna y representados en la forma que delimita el hilo en el aire, encerrando así una manera cultural de percibir el ambiente natural relacionándolo con la noción de cantidad.

El número 5, vicaic, el pez, con cinco partes delimitando la cabeza, el cuerpo y la cola del animal. La vida del pueblo qom tiene una íntima relación con el pez, base de su alimentación y fuente proteica que se come generalmente asado o al rescoldo o en preparaciones como sopas.

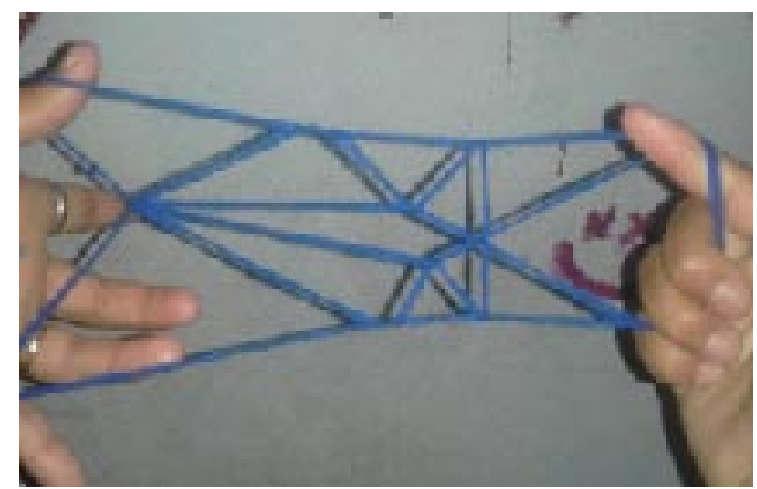

Figura 5: Vicaic-Pecesito- Amancia Silvestre

El número 6, cona, es la repetición de una forma, que vuelve a repetirse tantas veces como la idea de cantidad que muestra.

El número 7, naleguec, la red de pesca. La actividad de la pesca se realiza en forma compartida 


\section{Volumen 2 \\ "Semiótica y Narrativas"}

\section{Artículo}

Revista del grupo de

Estudios SEMTO-DISCURSNOS

y generalmente transcurren varios días en que los grupos familiares pasan a la vera de los ríos cercanos en la temporada cuando los peces abundan y sirven de alimentación para la comunidad, porque el hombre y la mujer qom solidariamente comparten los productos de la naturaleza.

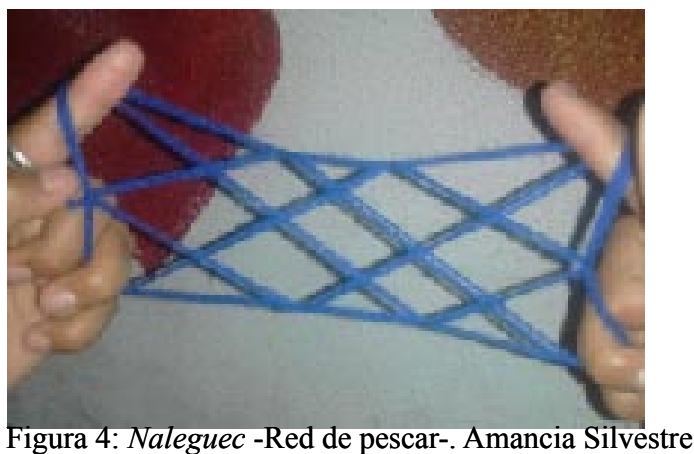

\section{Cona de la red Naleguec}

Margarita relata en qom mientras Juana realiza la Red -Naleguec-.

Juana: -Ella es mi madre, lo que ella comenta es que cona es un juego, simplemente un juego, pero deja muchas enseñanzas, porque a la vez que el chico juega el padre también le explica para qué sirven las redes. Para hacer esta red se trabaja la imaginación del chico, pero también aprende que la red significa mucho porque es algo que se realiza, se confecciona para poder pescar, para atrapar los peces. Esos peces se comparten con las familias y la comunidad. entonces todos tienen alimento en el día.

El número 8, nqo olliguishec, el caramelo. Podemos advertir que en un tiempo cercano en la historia se fue incorporando en el contacto con la cultura blanca esta golosina que es deseada por los asombrados pequeños que desconocían este producto dulce. En la relación luego de la conquista y la civilización europea comienzan a incorporar en su representación numérica figuras como la del caramelo, entre las familias con hijos e hijas pequeños. Tradicionalmente el dulce apetecido como postre es la miel silvestre que se recoge en el monte y se guarda cuidadosamente en bolsas de cuero donde se conserva para degustarla con harina de algarroba o de otras plantas.

\section{Sonia:}

-Los momentos que nosotros jugábamos es más que nada para entretenernos a la noche, por ejemplo. Esto es importante para enseñar en el nivel inicial porque en el tiempo libre, cuando los niñitos no tienen otra cosa que hacer, jugamos a esto y se entretienen. Ayuda mucho a la imaginación. Ahora sí hay docentes qom que juegan en la escuela y los chicos se entretienen. Antes sólo jugábamos en nuestra casa. La maestra qom es la que lleva los hilos y pueden compartir con niños criollitos. En la actualidad hacemos el cona del caramelo, antes no existía porque no comíamos caramelos.

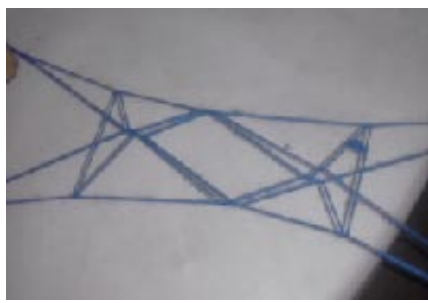

Figura 3: Nqo'oilliguishec -. Caramelo-. Amancia Silvestre 
El número 9, cona, se advierte en la trama que forman los hilos nueve espacios de la misma figura en una sucesión para enseñar etnomatemática y dar la idea de numero o cantidad.

El número 10, nala, es el sol, el astro que da la vida y la sostiene, el hermoso modo que usan los qom para representar la alegría de la luz. Es una figura que también se puede realizar entre varias personas, un juego de destreza y habilidad compartida y que consolida las tareas que aúnan los lazos de quienes emprenden juntos el camino. Grupos de mujeres o madres e hijas mueven los dedos con agilidad, conversan, mientras se mezclan las voces con el sonido de las risas de los jugadores y los ojos contemplan felices la gran figura central de un sol que alumbra con sus rayos a quienes habitan el territorio.

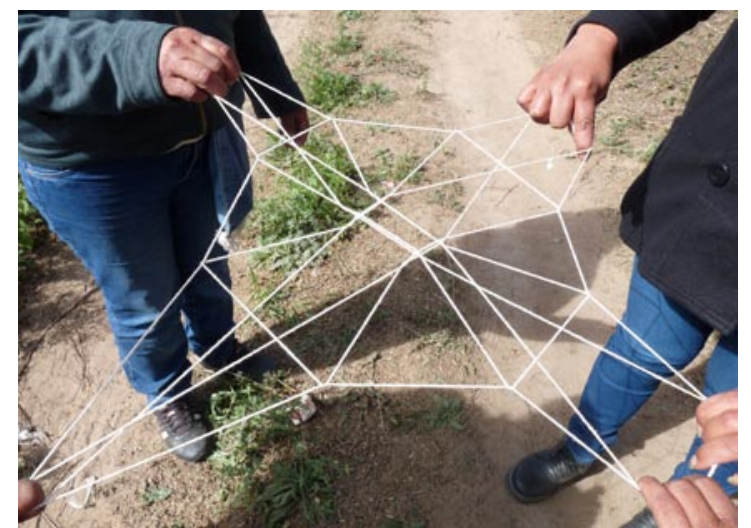

Figura: Nalá -Sol-Trabajo de campo con las MCCQ. 2018. Pampa del Indio.

Dice Victoria, que antes cuando no había relojes, los antiguos se guiaban por el horario del sol. El sol trae señas o avisos y un ejemplo es que cuando el sol si está rodeado por círculos como el color del arco iris, dice que vienen los meses lluviosos. Otro era que cuando sale el sol con las patitas o entra con las patitas, es una señal de que el clima viene también muy peligroso con tormentas. Otra cosa que había visto antiguamente era que el sol cuando se pone en el medio indica las doce en punto y se guiaban por las sombras del sol. El juego del hilo sin fin también se hace con la figura del sol. Es el juego que además de divertirse en grupo ayuda a tener habilidad en las manos, coordinación visomotora y ayuda a valorizar la memoria. Sí, porque vas pensando en los procesos y cómo armar el sol o también otras figuras con el hilo sin fin. Para la cultura toba el sol es el astro rey que da vida a toda la naturaleza.

El cielo es un pizarrón donde los grandes mensajes se pueden leer con solo levantar los ojos, en el lenguaje cifrado de los astros y sus colores, sus signos que al ser conocidos e interpretados anuncian los cambios de estaciones o las características del tiempo. Siempre hay una oportunidad para compartir, para desafiar a la creatividad con un nuevo intento de plasmar las formas en el tapiz invisible que arman los dedos de las manos entre los hilos de chaguar, que se transforman en pequeños lápices con los que ir creando un lenguaje milenario de generaciones que se comunican culturalmente desde la riqueza de sus símbolos y formas.

En este proceso de jugar y crear a través del juego, no sólo se conserva la memoria sino que se cruzan umbrales, se atraviesan fronteras culturales y se ponen en contacto las lenguas, las costumbres y la memoria ancestral del pueblo Qom con la cultura criolla. La narración de las historias que tejen los dedos experimentados de las madres, se convierte en un puente que pone en contacto semiosferas diferentes abriendo el diálogo intercultural. 


\section{Volumen 2 \\ "Semiótica y Narrativas"}

\section{Artículo}

Revista del grupo de

Estudios SEMIO-DISCURSNOS

El juego del hilo sin fin -Cona- fluye con la rítmica lúdica, empujada por fuerzas creativas y placenteras que trasuntan belleza en las formas de un lenguaje. La belleza es un valor intrínseco a todas las culturas en todos los tiempos y espacios. Su sentido cambia con la dinámica de las transformaciones sociales. La etnomatemática se posiciona como un espacio de diálogo intercultural con múltiples memorias. Eco (2002) en su ensayo sobre la Historia de la Belleza advierte que Pitágoras en el s. VI a.C. comienza a buscar relaciones entre la cosmología, la matemática, las ciencias naturales y la estética. También en la arquitectura la matemática aportará la proporción y la geometría, siendo el Tetratkys la figura simbólica de la conversión de lo numérico a lo espacial, y de lo aritmético a lo geométrico. Esta figura demuestra que el 10 resulta de sumar $1+2+3+4$, o sea, que es la suma de los cuatro primeros números enteros.

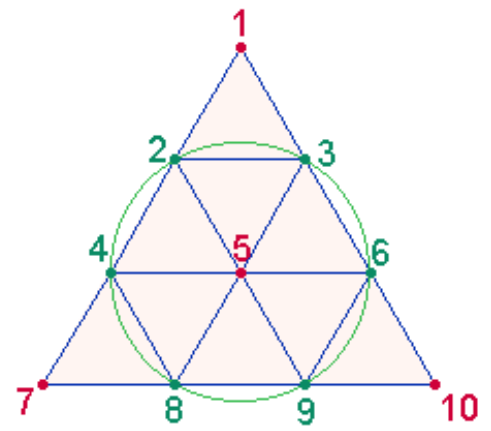

Tetratkys o década

http://latetraktys.blogspot.com/2010/03/v-behaviorurldefaultvml-o_07.html

Para Eco, a estas armonías aritméticas le corresponden también armonías geométricas y el ojo podrá enlazar constantemente estos puntos en una serie indefinida y encadenada de triángulos equiláteros perfectos (2002: 64). De modo análogo la cultura Qom enlaza significados de Cona en Naleguec, Nalá, Nqo'olliguishec, Mañec, Chi' Ishe, Vivaic, entre otras, figuras geométricas, pero también con funciones aritméticas que constituyen un sistema de representación. Como el mundo griego, los Qom utilizaron y utilizan Cona para fines prácticos como para fines pragmáticos, este último está íntimamente ligado a la enseñanza de crianza de las MCCQ en educación bilingüe intercultural. Recuperar el sentido de las prácticas antiguas, aprender a deslizar los dedos por entre las fuertes hebras del chaguar, es un acto de soberanía y libertad, que se imprime en el cuerpo y el espíritu de quienes insisten en cuidar y valorar toda creación humana, en un camino de educación para la paz y la armonía.

\section{| 3. Cona: de la memoria a la historia en perspectiva intercultural}

Como en la memoria de papel (Eco:2005) que pudo pertenecerle al tiempo de cualquier persona, las imágenes de figuritas, sellos, almanaques, revistas, entre otras formas que aparecen en recuerdos que no terminan de delinearse, así también las incontables formas de Coná entretejen memorias del hilo.

En perspectiva intercultural relatos y Cona van juntos, las formas habladas narran el pasado. En tal sentido, Cona escribe las palabras del pasado. Dicho esto, podemos reconocer atributos que comprenden la memoria social, dan carácter a la historia oral y constituyen elementos novedosos para el análisis histórico en esta perspectiva, a saber: 


\section{Volumen 2 \\ "Semiótica y Narrativas"}

\section{Artículo}

Revista del grupo de

Estudios SEMTO-DISCURSNOS

características de consideración epistemológica en perspectiva intercultural. Se trata de la experiencia en la memoria-territorio vivido. (Sandoval, 2013:51)

-Las vivencias del tiempo se manifiestan en la "data", el recuerdo es el dato del pasado, objetivamente temporales. El hábito-memoria (Ricoeur, Loc. Cit.) se "traduce" en su configuración simbólica de memoria territorio- vivido, dada la vital relación de los qom con el entorno natural.

-Orienta el análisis hacia la reflexión intercultural sobre las fuentes que legitiman los recuerdos en el acto de historizar el pasado, generalmente desestimadas por el discurso histórico hegemónico, lo que expresa una asimetría entre los argumentos de la memoria indígena y los argumentos de la memoria del blanco.

-Tiene carácter testimonial en tanto la verosimilitud, reconocimiento y respeto de quienes relatan y consistencia para el análisis veritativo. Son vivencias del tiempo y contienen verdades apriorísticas, pertenecientes a momentos constitutivos de la objetividad (Ricoeur, 2008: 52),

-Es una fuente de la memoria social, en tanto forma tangible retiene y representa en su atributo de -ficción/imagen- la huella del pasado. La forma es la "aprehensión de ahora", "un recuerdo primario" de la memoria-territorio-vivido (Sandoval, 2018:83).

-Desarrolla un proceso de trabajo que va de la reproducción verbal a la producción e invención de un esquema material -simbólico-, que actúa organizando e impulsando la coherencia del relato. El símbolo, como imagen, se acomoda como analogías del relato en las formas del -hilo- Cona.

- Los relatos que acompañan a Cona tienen sentido moralizante, "el niño aprende que la red significa mucho porque se confecciona para poder pescar, para atrapar los peces. Esos peces se comparten con las familias y la comunidad. entonces todos tienen alimento en el día". (Margarita, ut supra)

-Nuevas formas del pasado reciente dan cuenta de procesos de asimilación. Cona Nqo 'oilliguishec -. Caramelo- expresa la dinámica del cambio cultural en la experiencia histórica de contactos entre las culturas.

Se plantea así una primera cuestión para la reflexión en torno a la estabilización de las prácticas culturales que en el uso cotidiano y mediante la dinámica de las interacciones densifican y expresan lo que se reconoce como identidad cultural.

Los qom habitan la identidad cultural entre lo heredado y lo novedoso introducido en la dinámica histórica de las relaciones interculturales. Esto ocurre al mismo tiempo que pueden presentarse relaciones de resistencia y/o asimilación a las que históricamente se vieron expuestos. Interesa aquí poner de relieve algunas de las expresiones que dan cuenta de la habitabilidad de la cultura en los procesos de cambio, entre las cuales lo heredado y lo nuevo interactúan, sin que por ello se disuelvan los rasgos de identidad. (Sandoval, 2018:189).

La memoria social recupera en el juego del hilo sin fin -cona- usos y costumbres qom, las prácticas de maternaje reproducen y conservan la cultura qom y la lengua viva. Los relatos de las MCCQ sobre los significados del Cona sacan a la luz experiencias que fueron y son parte de la realidad social de la comunidad qom y los presentan con la fuerza creativa de la imagen. La palabra hablada proveniente de los relatos y Cona forman una fuente entrelazada para la historia oral en la que el símbolo y la narración son inseparables. 


\section{Volumen 2 \\ "Semiótica y Narrativas"}

\section{Artículo}

Revista del grupo de

Estudios SEMTO-DISCURSNOS

de maternaje y el valor que adquiere como práctica política. Las Madres Cuidadoras de la Cultura Qom, si bien conciben las prácticas de maternaje en el ámbito de la crianza doméstica de la familia extensa, en su carácter de organización étnica, instituyen estas prácticas para relacionarse interculturalmente con la cultura hegemónica. Las MCCQ introdujeron el juego del cona en los jardines y en las escuelas. Cona tiene el valor de bien patrimonial de la cultura y así lo utilizan. En este plano la memoria y la historia oral adquieren un fin práctico para la vida política, expresa los testimonios de los sin voz (Jelin,2002: 89)

La labor de transmisión de la memoria colectiva de las MCCQ a través de las prácticas de maternaje, configura en traducción intercultural, la oportunidad para comprender y ejercitar la práctica emancipatoria desde el saber pedagógico idóneo, en tanto expresa como ha devenido la CQPI y su posicionamiento en el presente, en el que la memoria activa del saber pedagógico adquiere así el valor moral y ético de reconstrucción y reconocimiento del pasado. (Sandoval, 2018:154)

Desde el 2003 la organización MCCQ inicia un proceso de reparación y restitución de derechos conculcados, labor que visibiliza la importancia del género en las memorias (Jelin, Op. Cit., pág. 99). Las MCCQ son historiadoras de su propio pasado. El derecho a la identidad y pertenencia están presentes en Cona, fuente histórica de la lengua qom, testimonio de lugares comunes, ríos, monte, animales, plantas, constelaciones. Tal como se mencionó más arriba, la narración genera comunidad, socialización y conservación de la memoria. Asimismo, en el caso del juego Cona, en el que se anudan relatos ancestrales, establece un puente, cruza una frontera hacia otra semiosfera (Lotman) desplegando un proceso de traducción entre distintos espacios de sentido: la cultura qom y la cultura criolla.

Descripciones de hechos que caracterizan y describen un territorio habitado hace cientos de años, ante de la conquista y de la colonización etnocida del blanco. Resulta insoslayable para la formación de grado redoblar esfuerzos en la educación intercultural en contextos sociales con pueblos indígenas. Los aportes interdisciplinares tienen la oportunidad única de recrear los contenidos del currículo e innovar a partir de una enseñanza compartida, abriendo espacios de participación a las y los integrantes de las comunidades indígenas.

Jugando con el hilo, Cona y la memoria que se evoca, elaboran un "proceso de recuperación para volver a dibujar el pasado. Recuerdos llenos de significados, incógnitas por descubrir. Cada narración habla de cosas nuevas (Eco:2005), de un mundo que ha sido y es el mundo de la cosmovisión de las MCCQ, pero también el mundo de lazos con otros universos culturales que llegan al juego y esperan ser narrados.

\section{Referencias bibliográficas}

Butler, J. (2017). Política de género y el derecho a aparecer, Cuerpos aliados y lucha política. Hacia una teoría performativa de la asamblea. (p.p. 68-69), Paidós, Buenos Aires.

Eco, U. (2005). Una memoria de papel. La misteriosa llama de la reina Loana. 95-105. Lumen. Bs. As.

(2002). III. La belleza como proporción y armonía. Historia de la Belleza. Extraído de: https://tallerdelaspalabrasblog.files.wordpress.com/2016/04/eco-umberto-historia-de-la-belleza.pdf el $05 / 09 / 21$. 


\section{Volumen 2 \\ "Semiótica y Narrativas"}

\section{Artículo}

Revista del grupo de

Estudios SEMIO-DISCURSNOS

Fornet Betancourt, R. (2005) Supuestos, límites y alcances de la Filosofía Intercultural. $V^{\circ}$ Congreso de Filosofía Intercultural. págs. (p.p. 261-274). Aache, Alemania, Brocar y Missionwissenschafliches Institut.

Gómez, Cecilia Paula y Braunstein José (2020), Cielo y juegos de hilo. Representación de la temporalidad cíclica entre los pilagá del Pilcomayo. Dossier "Contribuciones antropológicas a la comprensión del pasado en el Gran Chaco sudamericano" Dossier Editores invitados: Guillermo Lamenza, Luis del Papa \& Graciela Bailliet. Revista del Museo de la Plata, 2020, Volumen 5, Número 2: (p.p.602-617). Extraído de: https://publicaciones.fcnym.unlp.edu.ar/rmlp/article/view/2424/1982 el 18/05/21

Inda, Cristina (2013): Relación con el entorno natural. Uso cultural de las plantas. Historias de vida. Registro etnobotánico. "Experiencias en Educación Bilingüe Intercultural con las Madres Cuidadoras de la Cultura Qom”. págs. 80-86. Facultad de Humanidades, Resistencia. UNNE. Contexto.

Jelin, E., (2002). Trauma, testimonio y verdad. Los trabajos de memoria. págs. 79-90. Siglo XXI, Argentina.

(2002). El género en las memorias. Págs. 99-103. Ibid.

López L. E., (2015). El hogar, la comunidad y la escuela en la revitalización de las lenguas originarias de América Latina. En: Pueblos indígenas y educación, Julio-Diciembre $N^{\circ}$ 64, P.p. 211- Ediciones Abya-Yala, Quito-Ecuador.

Huizinga, J. (1972). 1. Esencia y significación del juego como fenómeno cultural. Homo ludens. (págs. 11-12). Alianza, Madrid.

-(1972). El concepto de juego y sus expresiones en el lenguaje. Homo ludens. (págs. 42-43).

Ibid.

García, M (2004) Narración. Semiosis/memoria. Posadas, Misiones, Editorial Universitaria UNaM. $2^{\mathrm{a}}$ ed.

Lotman, I.. (1996) La semiosfera I. Semiótica de la cultura y el texto. Madrid, Cátedra.

Peirce, C. (1987). Obra Lógico -semiótica. Madrid: Taurus.

Ricoeur, P. (2008). Memoria e imaginación. La memoria, la historia, el olvido. (p.p..48-49). Fondo de Cultura Económica. Argentina.

Sandoval, S. (2013). Las prácticas de maternaje de las Madres Cuidadoras de la Cultura Qom. "Experiencias en Educación Bilingüe Intercultural con las Madres Cuidadoras de la Cultura Qom". (p.p. 50-51). Facultad de Humanidades, Resistencia. UNNE. Contexto.

Memoria y territorio vivido "Experiencias en Educación Bilingüe Intercultural con las Madres Cuidadoras de la Cultura Qom”. (p.p.. 51-59). Facultad de Humanidades, Resistencia. UNNE. Contexto.

Sandoval, S., (2018) Reconstrucción de la memoria histórica de la comunidad Qom de Pampa del Indio. 


\section{Volumen 2 \\ "Semiótica y Narrativas"}

\section{Artículo}

Revista del grupo de

Estudios SEMTO-DISCURSNOS

Enfoque intercultural. 8. El plano simbólico de la identidad en perspectiva intercultural. (p. 189). Tesis doctoral. Fecha de la defensa 14/11/2018. En: https://repositorio.unne.edu.ar/handle/123456789/9041

\section{| Audiovisual}

Madres Cuidadoras de la Cultura Qom, Sandoval, Sylvia, Inda Cristina (2015). Ciclo del Cháguar y Cona: juego del hilo sin fin. (documental) ISBN 978-987-3619-05-2 Corrientes, Argentina. Universidad Nacional del Nordeste. Facultad de Humanidades. Extraído de: https://www.youtube. com/watch?v=GB gBF6UqBw

Entrevista

Madres Cuidadoras de la Cultura Qom (2018), Entrevista realizada en agosto en el Salón de las MCCQ, Pampa Chica, Pampa del Indio, Chaco, Argentina. Proyecto de Investigación 2015-2018- "Las Prácticas de Maternaje Qom y su aporte a la formación de grado en Educación Bilingüe Intercultural en contextos sociales con Pueblos Indígenas. Tercera Etapa". Resol. No 984/14 (10-diciembre) C.S. Secretaría General de Ciencia y Técnica. UNNE. Entrevistada por Sylvia E. Sandoval. 